\title{
Association of Toll-Like Receptor 4 Gene Polymorphisms with Acute Aortic Dissection in a Chinese Han Population
}

\author{
Tan Li $\mathbb{D},{ }^{1}$ Xiaozheng Liu $\mathbb{D}^{1},{ }^{1}$ Hongxia Ning $\mathbb{D},{ }^{1}$ Xintong Li $\mathbb{D},{ }^{2}$ Jun Yang $\mathbb{D},{ }^{1}$ \\ and Chunyan $\mathrm{Ma} \mathbb{( i )}^{1}$ \\ ${ }^{1}$ Department of Cardiovascular Ultrasound, The First Hospital of China Medical University, Shenyang 110001, China \\ ${ }^{2}$ Department of Vascular and Thyroid Surgery, The First Hospital of China Medical University, Shenyang 110001, China \\ Correspondence should be addressed to Chunyan Ma; cmulh_mcy@126.com
}

Received 24 August 2020; Revised 23 November 2020; Accepted 26 November 2020; Published 9 December 2020

Academic Editor: Cynthia C. Cardoso

Copyright (C) 2020 Tan Li et al. This is an open access article distributed under the Creative Commons Attribution License, which permits unrestricted use, distribution, and reproduction in any medium, provided the original work is properly cited.

\begin{abstract}
Background. Inflammation may be involved in the pathogenesis of acute aortic dissection (AAD). Toll-like receptor 4 (TLR4) is known to play a critical role in regulating the immune and inflammatory processes. To date, the relationship between genetic variation of TLR4 and AAD is far from clear. The purpose of our study was to illustrate the relevance of TLR4 polymorphisms with the susceptibility to AAD. Methods. A total of 222 AAD patients and 222 controls were enrolled in this study. Frequency distributions of TLR4 polymorphisms (rs10759932 in the promoter and rs11536889 in the $3^{\prime}$-untranslated region) were determined by the KASP method. Clinical parameters were acquired from subjects' medical records, and serum TLR4 levels were collected from our previously published data. Results. We found that rs10759932 polymorphism was associated with a reduced risk of $\mathrm{AAD}$ in the overall population (CC vs. TT: $\mathrm{OR}=0.393,95 \% \mathrm{CI}=0.164-0.939, P=0.036$; recessive model: $\mathrm{OR}=$ $0.439,95 \% \mathrm{CI}=0.196-0.984, P=0.045)$ and subgroup analyses stratified by sex. The GC genotype and dominant model of rs11536889 conferred a significantly higher risk of AAD compared with GG genotype in female subjects (GC vs. GG: OR= 3.382, 95\%CI $=1.051-10.885, \quad P=0.041 ;$ dominant model: $\mathrm{OR}=3.043,95 \% \mathrm{CI}=1.041-8.900, \quad P=0.042)$. In addition, a significant interaction between the rs11536889 recessive model and dyslipidemia was observed for an increased risk of AAD $\left(P_{\text {interaction }}=0.038, \mathrm{OR}=15.229\right)$ after the adjustment for potential clinical covariates. We also used the false-positive report probability (FPRP) analysis to validate the significant results. Furthermore, rs11536889 polymorphism could affect the maximal aortic diameters of $\mathrm{AAD}(P=0.037)$, while AAD patients carrying CC genotype of rs 10759932 showed lower serum TLR4 levels than TT genotype carriers $(P=0.043)$. Conclusions. Our findings provide evidence for the association between TLR4 polymorphisms and AAD susceptibility in a Chinese Han population, which may have some implications for understanding the role of TLR4 in the pathophysiology of AAD.
\end{abstract}

\section{Introduction}

Acute aortic dissection (AAD) is the most life-threatening macrovascular disorder with high mortality, characterized by intimal tear and surging of blood into the medial layer of the aorta $[1,2]$. The etiology of AAD is complex and heterogeneous. In addition to some environmental risk factors, such as smoking, male, old age, hypertension, and dyslipidemia, genetic factors are also viewed to contribute to the pathogenesis of $\mathrm{AAD}[3,4]$. Although the responsible molecular and genetic determinants of AAD remain largely unidentified, it has been widely accepted that inflammation plays an essential role in the structural damage of the aortic wall and the development of AAD [2, 5, 6]. Increasing data indicated that elevated inflammatory cell infiltration and higher expression levels of inflammatory mediators, including C-reactive protein (CRP) and D-dimer, were detected in dissected aortic specimens and peripheral blood of $\mathrm{AAD}$ 
patients [7-9]. More importantly, the local inflammation in the aortic wall and subsequent systemic inflammatory reaction were observed during the whole course of $\mathrm{AAD}[2,10$, 11]. As a consequence, investigating the inflammationrelated pathogenic genes would be beneficial to understand the underlying mechanisms of AAD and to prevent and treat the disease.

Toll-like receptor 4 (TLR4), located on chromosome $9 \mathrm{q} 32-\mathrm{q} 33$, is not only the key pattern-recognition receptor in immune-inflammatory reactions but also the initiation protein in this signal transduction pathway [12]. Recently, the role of TLR4-mediated signaling has emerged in maintaining aortic homeostasis and establishing the aorta alterations (vascular remodeling and medial degeneration) and their complications $[13,14]$. It was confirmed that the upregulation of TLR4 could evocate inflammatory cell infiltration, production of proinflammatory mediators, endothelial dysfunction, smooth muscle cell apoptosis, and aortic media degradation, which were closely related to aortic inflammation, remodeling, and dissection [15-17]. In our previous study, we preliminarily found that an elevated level of serum TLR4 expression was independently associated with the risk of $\mathrm{AAD}$, and there was a positive relationship between serum TLR4 and circulating CRP [18]. The activity and function of TLR4 seem to be modulated by genetic variations, principally single nucleotide polymorphisms (SNPs), which may change the ligand binding and balance between pro- and antiinflammatory cytokines, thereby regulating the progression of various diseases $[19,20]$. Therefore, it is reasonable to hypothesize that SNPs in the functional regions of the TLR4 gene may have effects on TLR4 activity and thus modify the signaling of immune and subsequent inflammatory responses, which in turn may affect AAD risk. Some evidences have exhibited that TLR4 polymorphisms were closely associated with the risk of infection [21], atherosclerotic disease [22], autoimmune disease [23], and tumor [24]. One study revealed that polymorphisms linked to the TLR4-mediated metalloproteinase pathways could obviously impact the risk of sporadic thoracic aortic aneurysm [25]. It remains unclear, however, whether TLR4 polymorphisms are relevant to the susceptibility of AAD.

In this case-control study, we aimed to discuss the correlation between TLR4 polymorphisms and AAD risk and examine whether potential gene-environment interactions could enhance the susceptibility to AAD. In addition, the consequences of these SNPs on the levels of AAD-related parameters and serum TLR4 in AAD patients were further investigated. Our study might contribute to the prediction of genetic variants associated with disease risk and add knowledge for the prevention and treatment for AAD.

\section{Material and Methods}

2.1. Study Population. A total of 222 AAD patients and 222 controls were enrolled from the First Hospital of China Medical University from May 2017 to August 2018. Case and control participants were all Chinese Han population and matched by age and sex. All the patients were evaluated within 24 hours after symptom and diagnosed by the com- puted tomography angiography (CTA), and there were 172 patients under surgical treatment. Excluding criteria incorporated the subjects with coronary heart diseases, congenital cardiovascular defects, severe vascular stenosis, autoimmune diseases, severe organ failure, infectious diseases, hematological system diseases, or malignant tumors. Patients with certain genetic syndromes, such as Marfan syndrome and Ehlers-Danlos syndrome, or traumatic aneurysms were also excluded from the study. A $5 \mathrm{~mL}$ fasting venous blood sample was obtained from each subject for DNA isolation. The study was approved by the Ethics Committee of the First Hospital of China Medical University (Shenyang, China). Written informed consent was obtained from each participant.

2.2. Data Collection. The demographic data and clinical related information were collected from participants' medical records. And maximal aortic diameters of AAD subjects were assessed by CTA. Smoking was defined as having smoked at least one cigarette per day for more than one year. Drinking was defined as having consumed at least one alcoholic drink a day for a minimum period of six months. Body mass index (BMI) was computed as weight in kilograms divided by the square of height in meters. Obesity was defined as BMI $\geq 28$ $\mathrm{kg} / \mathrm{m}^{2}$. Hypertension was defined as systolic blood pressure $(\mathrm{SBP}) \geq 140 \mathrm{mmHg}$ and/or diastolic blood pressure (DBP) $\geq 90 \mathrm{mmHg}$ and/or use of antihypertensive medications. Diabetes was defined as fasting plasma glucose $(\mathrm{FPG}) \geq 7$ $\mathrm{mmol} / \mathrm{L}(126 \mathrm{mg} / \mathrm{dL})$ and/or under antidiabetic treatment. Dyslipidemia was defined as total cholesterol $(\mathrm{TC}) \geq 6.22$ $\mathrm{mmol} / \mathrm{L}(240 \mathrm{mg} / \mathrm{dL})$, or triglyceride $(\mathrm{TG}) \geq 2.26 \mathrm{mmol} / \mathrm{L}$ (200 mg/dL), or high-density lipoprotein cholesterol (HDLC) $<1.03 \mathrm{mmol} / \mathrm{L}(40 \mathrm{mg} / \mathrm{dL})$, or low-density lipoprotein cholesterol $(\mathrm{LDL}-\mathrm{C}) \geq 4.14 \mathrm{mmol} / \mathrm{L} \quad(160 \mathrm{mg} / \mathrm{dL}) \quad$ and/or under taking hypolipidemic drugs.

2.3. SNP Selection and Genotyping Assay. A two-step approach was adopted to identify tag-SNPs in TLR4 [26]. Briefly, we applied the combination of HapMap database (http://www.HapMap.org) and Haploview software 4.2 (http://www.broadinstitute.org/mpg/haploview) to select the tag-SNPs, which should fit the following criteria: minor allele frequency $(\mathrm{MAF})>0.05$ in Chinese Han population, low linkage disequilibrium $\left(r^{2}<0.8\right)$, and Hardy-Weinberg equilibrium $(\mathrm{HWE})>0.05$. Then, potential functions of tag-SNPs were predicted with SNPinfo Web Server (https:// snpinfo.niehs.nih.gov/). Accordingly, rs10759932 in the promoter region and rs11536889 in the $3^{\prime}$-untranslated region ( $3^{\prime}$-UTR) of TLR4, which could separately modify the function of transcription factor binding sites and miRNA binding sites, were chosen in this study.

A routine phenol-chloroform method was utilized to extract genomic DNA from each blood clot. All samples were randomly placed on the 384-well plates and blinded for disease status. SNPs were genotyped by Baygene Biotechnology Company Limited (Shanghai, China) with the KASP method using the SNPLine platform (LGC, United Kingdom). Genotyping quality was assessed by repeated detection of $10 \%$ randomly selected samples, yielding a $100 \%$ concordance. 
2.4. SNP-Gene Expression Correlation Analysis. Based on our previously published data [18], a total of 64 AAD patients with the information of serum TLR4 levels were involved in further genotype and TLR4 gene expression correlation analysis.

2.5. Statistical Analysis. All the data analyses were conducted with SPSS 17.0 software (SPSS Inc., Chicago, IL, United States). HWE for studied SNPs in each group was evaluated with the chi-square $\left(\chi^{2}\right)$ test. Differences of baseline characteristics between AAD patients and controls were compared by the independent-sample $t$-test or $\chi^{2}$ test as appropriate. Comparisons of continuous variables among different genotype groups were performed with one-way ANOVA. The association of SNPs with AAD risk was estimated by calculating odds ratios (ORs) and their 95\% confidence intervals (CIs) using multivariate logistic regression after adjusting the potential confounding factors. The log-likelihood ratio test was performed to evaluate the SNP-environment interaction by comparing the model that only involved the main effects with the full model also containing the interaction term. The Bonferroni correction was used to adjust $P$ values for multiple tests as needed. Moreover, the false-positive report probability (FPRP) was calculated to verify the significant results at different prior probability levels. First, we used the software NCSS-PASS version 11.0.7 (USA) to test the statistical power of each association. Then, the FPRP values were figured out by following the published instructions, and only the significant result with FPRP $<0.5$ was regarded as a noteworthy finding [27]. A two-sided $P<0.05$ was considered statistically significant. In addition, the dominant and recessive genetic models were defined as heterozygote thomozygote variant vs. homozygote wild and homozygote variant vs. heterozygote+homozygote wild, respectively.

\section{Results}

3.1. Characteristics of the Study Population. Table 1 presents the baseline characteristics of the study participants. Compared with controls, AAD cases were not statistically different in age, sex, obesity, smoking, drinking, and dyslipidemia.

3.2. Association of TLR4 Polymorphisms with AAD Risk. The genotype distributions of rs10759932 and rs11536889 in each group are summarized in Table 2. The genotypes in controls were all in consistent with HWE $(P>0.05)$. After adjusting age, sex, obesity, smoking, drinking, hypertension, diabetes, and dyslipidemia, the rs10759932 CC genotype and recessive model were associated with a decreased risk of AAD with corresponding ORs of 0.393 (95\%CI $=0.164-0.939, P=$ $0.036)$ and $0.439(95 \% \mathrm{CI}=0.196-0.984, P=0.045)$, respectively. The overall genetic effects for rs11536889 related to AAD were not observed.

To explore the correlation between TLR4 polymorphisms and $\mathrm{AAD}$ risk in specific subgroups, we further conducted stratified analyses on the basis of age and sex, as shown in Table 3. For rs10759932, the recessive model was associated with a reduced AAD risk in male subjects $(\mathrm{OR}=0.343,95 \%$ $\mathrm{CI}=0.133-0.882, P=0.026)$, and the heterozygote $\mathrm{TC}$ and dominant model conferred a decreased risk of $\mathrm{AAD}$ in
TABLE 1: Baseline characteristics of the study participants ${ }^{\mathrm{a}}$.

\begin{tabular}{|c|c|c|c|}
\hline Variables & $\begin{array}{c}\text { Control } \\
(n=222)\end{array}$ & $\begin{array}{c}\text { AAD } \\
(n=222)\end{array}$ & $P$ \\
\hline Age (years) & $56.7 \pm 11.8$ & $56.3 \pm 12.2$ & 0.769 \\
\hline Male, $n(\%)$ & $163(73.4 \%)$ & $164(73.9 \%)$ & 0.914 \\
\hline Obesity & & & 0.081 \\
\hline Yes, $n(\%)$ & $42(18.9 \%)$ & $54(24.3 \%)$ & \\
\hline No, $n(\%)$ & $173(77.9 \%)$ & $146(65.8 \%)$ & \\
\hline Missing, $n(\%)$ & $7(3.2 \%)$ & $22(9.9 \%)$ & \\
\hline Smoking & & & 0.089 \\
\hline Yes, $n(\%)$ & $81(36.5 \%)$ & $73(32.9 \%)$ & \\
\hline No, $n(\%)$ & $112(50.4 \%)$ & $143(64.4 \%)$ & \\
\hline Missing, $n(\%)$ & $29(13.1 \%)$ & $6(2.7 \%)$ & \\
\hline Drinking & & & 0.065 \\
\hline Yes, $n(\%)$ & $71(31.9 \%)$ & $61(27.5 \%)$ & \\
\hline No, $n(\%)$ & $122(55.0 \%)$ & $155(69.8 \%)$ & \\
\hline Missing, $n(\%)$ & $29(13.1 \%)$ & $6(2.7 \%)$ & \\
\hline Hypertension & & & $<0.001$ \\
\hline Yes, $n(\%)$ & $93(41.9 \%)$ & $176(79.3 \%)$ & \\
\hline No, $n(\%)$ & $129(58.1 \%)$ & $46(20.7 \%)$ & \\
\hline Diabetes & & & $<0.001$ \\
\hline Yes, $n(\%)$ & $23(10.4 \%)$ & $82(36.9 \%)$ & \\
\hline No, $n(\%)$ & $199(89.6 \%)$ & $126(56.8 \%)$ & \\
\hline Missing, $n(\%)$ & - & $14(6.3 \%)$ & \\
\hline Dyslipidemia & & & 0.426 \\
\hline Yes, $n(\%)$ & $100(45.1 \%)$ & $105(47.3 \%)$ & \\
\hline No, $n(\%)$ & $121(54.5 \%)$ & $109(49.1 \%)$ & \\
\hline Missing, $n(\%)$ & $1(0.4 \%)$ & $8(3.6 \%)$ & \\
\hline WBC $\left(\times 10^{9} / \mathrm{L}\right)$ & - & $11.26 \pm 4.46$ & - \\
\hline $\mathrm{CRP}(\mathrm{mg} / \mathrm{L})$ & - & $77.00 \pm 60.88$ & - \\
\hline $\mathrm{D}$-dimer $(\mu \mathrm{g} / \mathrm{mL})$ & - & $4.65 \pm 4.49$ & - \\
\hline $\begin{array}{l}\text { Max. aortic diameter } \\
(\mathrm{cm})\end{array}$ & - & $4.48 \pm 0.95$ & - \\
\hline $\begin{array}{l}\text { Serum TLR4 levels } \\
(\mathrm{ng} / \mathrm{mL})\end{array}$ & - & $13.31 \pm 6.74$ & - \\
\hline
\end{tabular}

${ }^{\mathrm{a}}$ Demographic and clinical data for $222 \mathrm{AAD}$ patients and 222 controls were collected from the medical records, and maximal aortic diameters of all cases were assessed by CTA. And serum TLR4 levels in a total of 64 AAD subjects were obtained from our previously published data [18].

female subjects (TC vs. TT: $\mathrm{OR}=0.231,95 \% \mathrm{CI}=0.071$ 0.752, $P=0.015$; dominant model: $\mathrm{OR}=0.241,95 \% \mathrm{CI}=$ 0.082-0.707, $P=0.010)$. As for rs 11536889, its GC genotype and dominant model were significantly correlated with an increased risk of AAD in female subjects with $\mathrm{OR}$ values of 3.382 and 3.043 (all $P<0.05$ ), respectively, compared with GG genotype.

3.3. The Interactions between TLR4 Polymorphisms and Risk Factors in AAD Susceptibility. The interaction effect between TLR4 polymorphisms and environmental factors on the risk of AAD was examined. A combined genotype including the dominant and recessive genetic models of TLR4 SNPS was used for interaction analysis. Table 4 showed that the most 
TABLE 2: The association of TLR4 polymorphisms with the risk of AAD.

\begin{tabular}{|c|c|c|c|c|c|}
\hline & NCBI Ref & Control & $\mathrm{AAD}$ & $P\left(P_{\text {corr }}\right)$ & OR (95\% CI) \\
\hline rs10759932 & $n=28$ & $n=222$ & $n=222$ & & \\
\hline TT & $13(46.4 \%)$ & 93 (41.9\%) & $106(48.4 \%)$ & & \\
\hline TC & $12(42.9 \%)$ & $95(42.8 \%)$ & $94(42.9 \%)$ & 0.297 & $0.759(0.453-1.274)$ \\
\hline $\mathrm{CC}$ & $3(10.7 \%)$ & $34(15.3 \%)$ & $19(8.7 \%)$ & $0.036(0.072)$ & $0.393(0.164-0.939)$ \\
\hline CC+TC vs. TT & & & & 0.112 & $0.673(0.412-1.098)$ \\
\hline CC vs. TC+TT & & & & $0.045(0.090)$ & $0.439(0.196-0.984)$ \\
\hline$P_{\mathrm{HWE}}$ & & 0.239 & 0.775 & & \\
\hline rs11536889 & $n=84$ & $n=222$ & $n=222$ & & \\
\hline GG & $48(57.1 \%)$ & $154(70.3 \%)$ & $148(66.7 \%)$ & & \\
\hline GC & $31(36.9 \%)$ & $57(26.0 \%)$ & $58(26.1 \%)$ & 0.934 & $1.023(0.590-1.775)$ \\
\hline $\mathrm{CC}$ & $5(6.0 \%)$ & $8(3.7 \%)$ & $16(7.2 \%)$ & 0.598 & $1.351(0.441-4.135)$ \\
\hline $\mathrm{CC}+\mathrm{GC}$ vs. GG & & & & 0.818 & $1.063(0.634-1.780)$ \\
\hline CC vs. GC+GG & & & & 0.615 & $1.327(0.441-3.995)$ \\
\hline$P_{\mathrm{HWE}}$ & & 0.351 & 0.004 & & \\
\hline
\end{tabular}

$P$ for association was adjusted by age, sex, obesity, smoking, drinking, hypertension, diabetes, and dyslipidemia. NCBI Ref: reference frequencies of these SNPs in the Asian population (NCBI database). $P_{\text {corr }}: P$ values after Bonferroni correction. The results are in bold if $P<0.05$.

significant interaction was between the rs11536889 recessive model and dyslipidemia and associated with an increased risk of $\mathrm{AAD}\left(P_{\text {interaction }}=0.038, \mathrm{OR}=15.229\right)$, after the adjustment for age, sex, obesity, smoking, drinking, hypertension, and diabetes. However, there were no significant interactions between rs10759932 and risk factors in AAD susceptibility.

3.4. FPRP Results. Now that AAD is a relatively rare disease and there are limited studies concerning the association between gene polymorphism and AAD risk, we set 0.5 as the FPRP threshold [27]. It was shown that all of the significant findings for TLR4 rs11536889 polymorphism remained noteworthy at the prior probability of 0.25 or 0.1 (Table 5 ).

3.5. The Association of TLR4 Polymorphisms with Clinical Parameters and Serum TLR4 Levels in AAD Patients. Larger aortic diameters were observed in rs11536889 CC genotype carriers when compared to GG genotype carriers $(P=0.037)$ (Table 6). In addition, AAD subjects with CC genotype had significantly lower serum TLR4 levels than those with TT genotype for TLR4 rs10759932 $(P=0.043)$ (Table 6 and Figure 1).

\section{Discussion}

To our knowledge, no investigation has focused on the association between TLR4 polymorphisms and AAD susceptibility. The current study is the first report to identify the significance of TLR4 rs10759932 and rs11536889 polymorphisms and their interactions with environmental factors in the risk of $\mathrm{AAD}$, as well as their associations with AADrelated clinical parameters and serum TLR4 levels.

$\mathrm{AAD}$ is one of the severe and major health issues of aortic disease known to be caused by inflammation, which could destroy the aortic structure and eventually lead to the aortic wall dissection and rupture [28]. TLR4 is considered a useful marker for evaluating local inflammatory reaction and has attracted particular interest because of its important function in mediating vascular remodeling and injury $[15,17]$. As the most common form of genetic variation, SNPs in TLR4 functional regions might cause a dysfunction of TLR4 molecule and interfere with the host immunity and inflammation response, contributing to the risk of various diseases. The SNP rs10759932 locates in the promoter region of the TLR4 gene and may regulate the TLR4 expression level by influencing the binding affinity of transcription factors [29]. The T to C allele substitution of rs10759932 has been reported to be strongly associated with a reduced risk of malignant tumors $[29,30]$. Similarly, our results indicated a significant association of rs10759932 CC genotype and recessive model with a reduced risk of $\mathrm{AAD}$ in the overall population, and the favorable effect of rs10759932 polymorphism on AAD was also prominent in the subgroup analyses stratified by sex. The rs11536889 polymorphism is located in the centre of the 2818-bp TLR4 3'-UTR, where a genetic change can influence mRNA stability and translation efficiency [24, 31]. A laboratory study by Sato et al. revealed that a fragment of $3^{\prime}$-UTR containing the TLR4 rs11536889 G allele, but not the C allele, inhibited luciferase activity triggered by LPS or IL-6 possibly by binding to miRNAs in posttranscriptional regulation [32]. Several studies found that TLR4 rs11536889 CC genotype or $\mathrm{C}$ allele was correlated with an increased risk of coronary artery disease [12], gastric cancer [33], and hepatitis A virus infection [34]. In this research, we figured out that female individuals carrying rs11536889 GC genotype or dominant model were more susceptible to AAD compared with those with GG genotype. The above findings implied that TLR4 rs10759932 and rs11536889 could be genetic biomarkers and potential therapeutic targets for AAD.

$\mathrm{AAD}$ is a complex trait, and its susceptibility may be enhanced by a combined effect of genetic background and 
TABLE 3: Association of TLR4 polymorphisms with the risk of AAD stratified by age and sex.

\begin{tabular}{|c|c|c|c|c|c|}
\hline & Genotypes & Control & $\mathrm{AAD}$ & $P\left(P_{\text {corr }}\right)$ & OR $(95 \% \mathrm{CI})$ \\
\hline \multicolumn{6}{|c|}{ rs10759932 } \\
\hline \multicolumn{6}{|l|}{ Age } \\
\hline & TT & $50(42.4 \%)$ & $61(52.1 \%)$ & & \\
\hline & TC & $46(39.0 \%)$ & $45(38.5 \%)$ & 0.464 & $0.786(0.413-1.497)$ \\
\hline \multirow[t]{5}{*}{$>55 y$} & $\mathrm{CC}$ & $22(8.6 \%)$ & $11(9.4 \%)$ & 0.075 & $0.418(0.160-1.093)$ \\
\hline & \multicolumn{2}{|c|}{ CC+TC vs. TT } & & 0.153 & $0.644(0.353-1.177)$ \\
\hline & \multicolumn{2}{|c|}{ CC vs. TC+TT } & & 0.097 & $0.472(0.195-1.144)$ \\
\hline & TT & $43(41.3 \%)$ & $45(44.1 \%)$ & & \\
\hline & $\mathrm{TC}$ & $49(47.1 \%)$ & $49(48.0 \%)$ & 0.598 & $0.787(0.323-1.917)$ \\
\hline \multirow[t]{3}{*}{$\leq 55 y$} & $\mathrm{CC}$ & $12(11.5 \%)$ & $8(7.8 \%)$ & 0.240 & $0.275(0.032-2.371)$ \\
\hline & \multicolumn{2}{|c|}{$\mathrm{CC}+\mathrm{TC}$ vs. TT } & & 0.482 & $0.731(0.306-1.749)$ \\
\hline & \multicolumn{2}{|c|}{$\mathrm{CC}$ vs. TC+TT } & & 0.315 & $0.355(0.047-2.682)$ \\
\hline \multicolumn{6}{|l|}{ Sex } \\
\hline & $\mathrm{TT}$ & $66(40.5 \%)$ & $67(41.4 \%)$ & & \\
\hline & TC & $70(42.9 \%)$ & $83(51.2 \%)$ & 0.560 & $1.198(0.652-2.198)$ \\
\hline \multirow[t]{5}{*}{ Male } & $\mathrm{CC}$ & $27(16.6 \%)$ & $12(7.4 \%)$ & 0.061 & $0.373(0.133-1.049)$ \\
\hline & \multicolumn{2}{|c|}{$\mathrm{CC}+\mathrm{TC}$ vs. TT } & & 0.935 & $0.976(0.549-1.737)$ \\
\hline & \multicolumn{2}{|c|}{ CC vs. TC+TT } & & $0.026(0.052)$ & $0.343(0.133-0.882)$ \\
\hline & $\mathrm{TT}$ & $27(45.7 \%)$ & $39(68.4 \%)$ & & \\
\hline & $\mathrm{TC}$ & $25(42.4 \%)$ & $11(19.3 \%)$ & $0.015(0.030)$ & $0.231(0.071-0.752)$ \\
\hline \multirow[t]{3}{*}{ Female } & $\mathrm{CC}$ & $7(11.9 \%)$ & $7(12.3 \%)$ & 0.253 & $0.365(0.065-2.054)$ \\
\hline & \multicolumn{2}{|c|}{ CC+TC vs. TT } & & $0.010(0.020)$ & $0.241(0.082-0.707)$ \\
\hline & \multicolumn{2}{|c|}{ CC vs. TC+TT } & & 0.480 & $0.550(0.105-2.890)$ \\
\hline \multicolumn{6}{|c|}{ rs11536889 } \\
\hline \multicolumn{6}{|l|}{ Age } \\
\hline & GG & $83(70.9 \%)$ & $78(66.7 \%)$ & & \\
\hline & GC & $30(25.6 \%)$ & $29(24.8 \%)$ & 0.935 & $1.029(0.518-2.044)$ \\
\hline \multirow[t]{5}{*}{$>55 y$} & $\mathrm{CC}$ & $4(3.4 \%)$ & $10(8.5 \%)$ & 0.436 & $1.807(0.408-8.009)$ \\
\hline & \multicolumn{2}{|c|}{$\mathrm{CC}+\mathrm{GC}$ vs. GG } & & 0.702 & $1.134(0.596-2.159)$ \\
\hline & \multicolumn{2}{|c|}{ CC vs. GC+GG } & & 0.423 & $1.831(0.417-8.031)$ \\
\hline & GG & $71(69.6 \%)$ & $70(66.7 \%)$ & & \\
\hline & GC & $27(26.5 \%)$ & $29(27.6 \%)$ & 0.907 & $1.064(0.379-2.983)$ \\
\hline \multirow[t]{3}{*}{$\leq 55 y$} & $\mathrm{CC}$ & $4(3.9 \%)$ & $6(5.7 \%)$ & 0.962 & $1.055(0.116-9.595)$ \\
\hline & \multicolumn{2}{|c|}{$\mathrm{CC}+\mathrm{GC}$ vs. GG } & & 0.941 & $0.965(0.367-2.532)$ \\
\hline & \multicolumn{2}{|c|}{ CC vs. GC+GG } & & 0.848 & $1.226(0.153-9.824)$ \\
\hline Sex & & & & & \\
\hline & GG & $114(71.3 \%)$ & $115(70.1 \%)$ & & \\
\hline & GC & $41(25.6 \%)$ & $40(24.4 \%)$ & 0.384 & $0.746(0.386-1.442)$ \\
\hline Male & $\mathrm{CC}$ & $5(3.1 \%)$ & $9(5.5 \%)$ & 0.585 & $1.469(0.369-5.839)$ \\
\hline & & & & 0.534 & $0.822(0.444-1.524)$ \\
\hline & & & & 0.482 & $1.630(0.418-6.360)$ \\
\hline & GG & $40(67.8 \%)$ & $33(56.9 \%)$ & & \\
\hline & GC & $16(27.1 \%)$ & $18(31.0 \%)$ & $0.041(0.082)$ & $3.382(1.051-10.885)$ \\
\hline Female & CC & $3(5.1 \%)$ & $7(12.1 \%)$ & 0.590 & $1.729(0.236-12.673)$ \\
\hline & & & & $0.042(0.084)$ & $3.043(1.041-8.900)$ \\
\hline & & & & 0.786 & $1.313(0.183-9.437)$ \\
\hline
\end{tabular}

$P$ for association was adjusted by obesity, smoking, drinking, hypertension, diabetes, and dyslipidemia. $P_{\text {corr }} P$ values after Bonferroni correction. The results are in bold if $P<0.05$. 


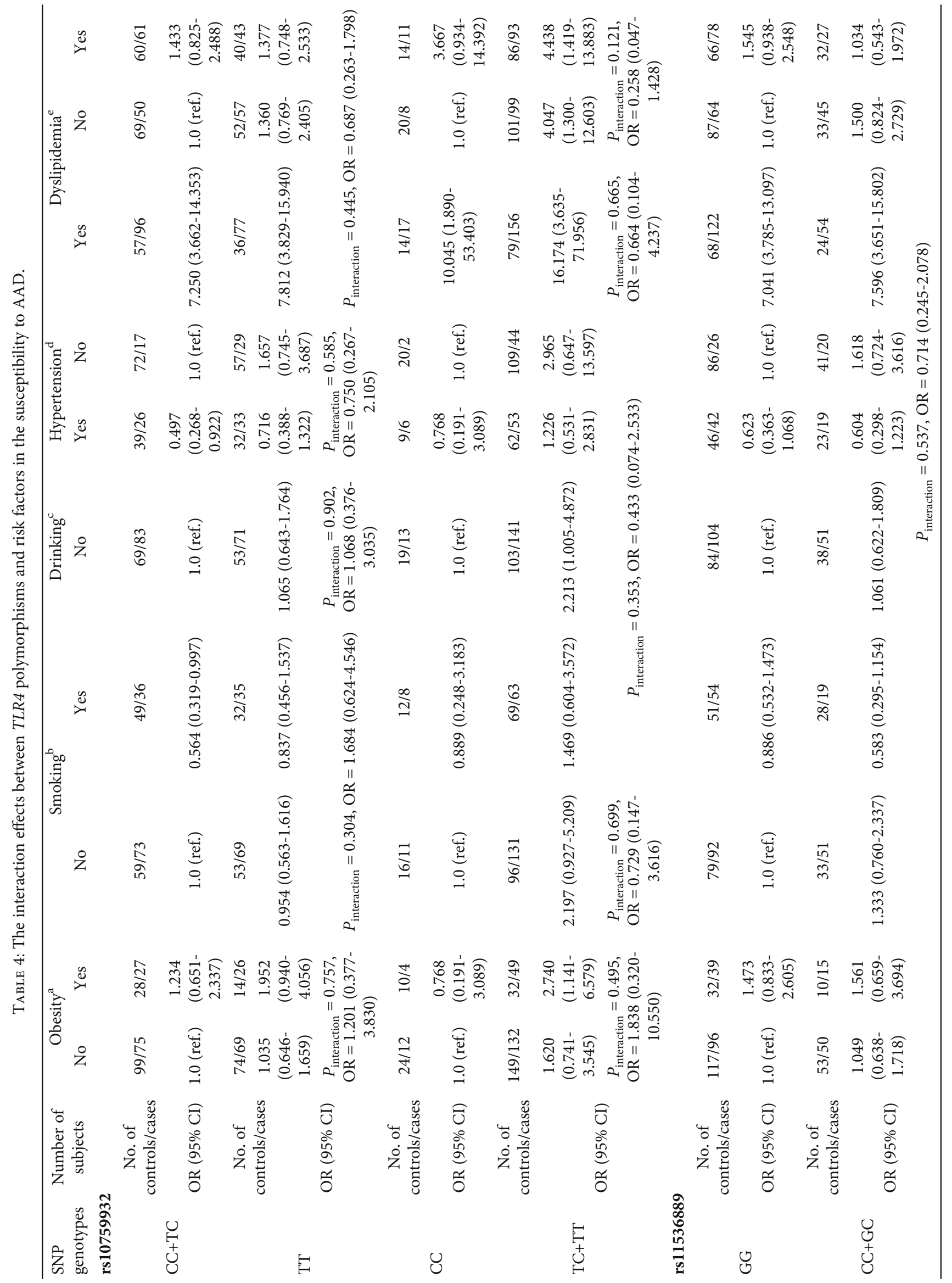




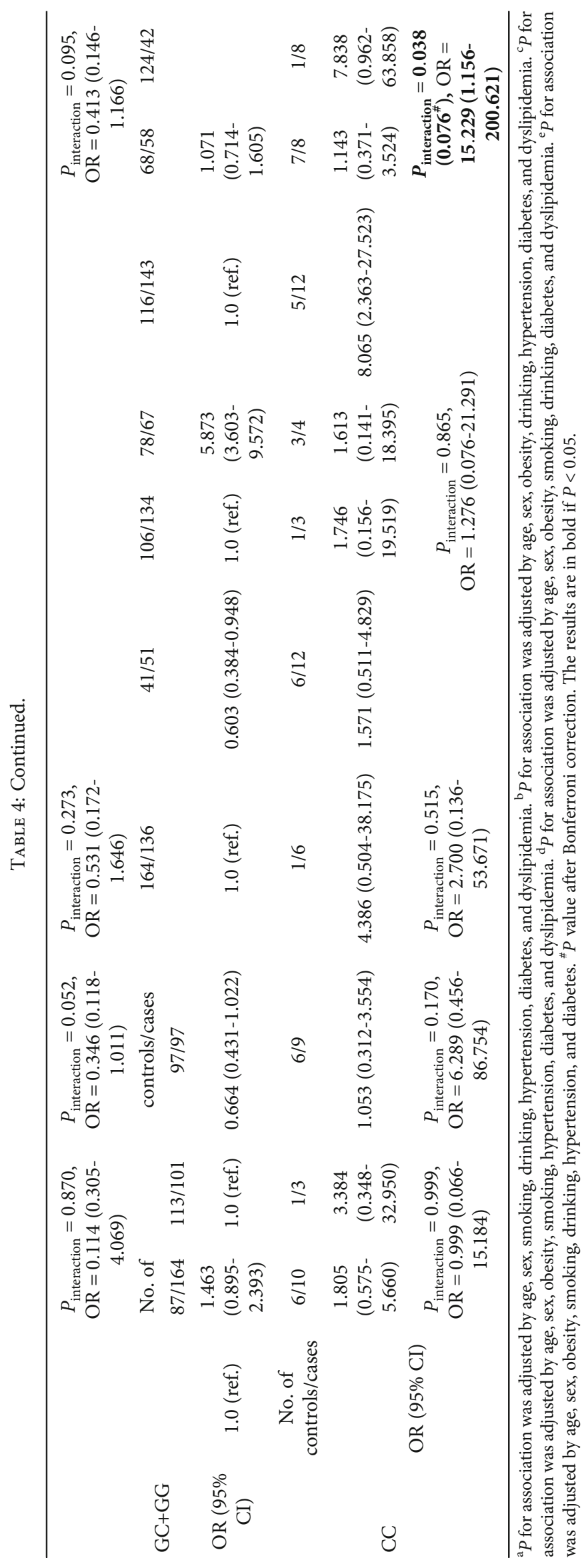




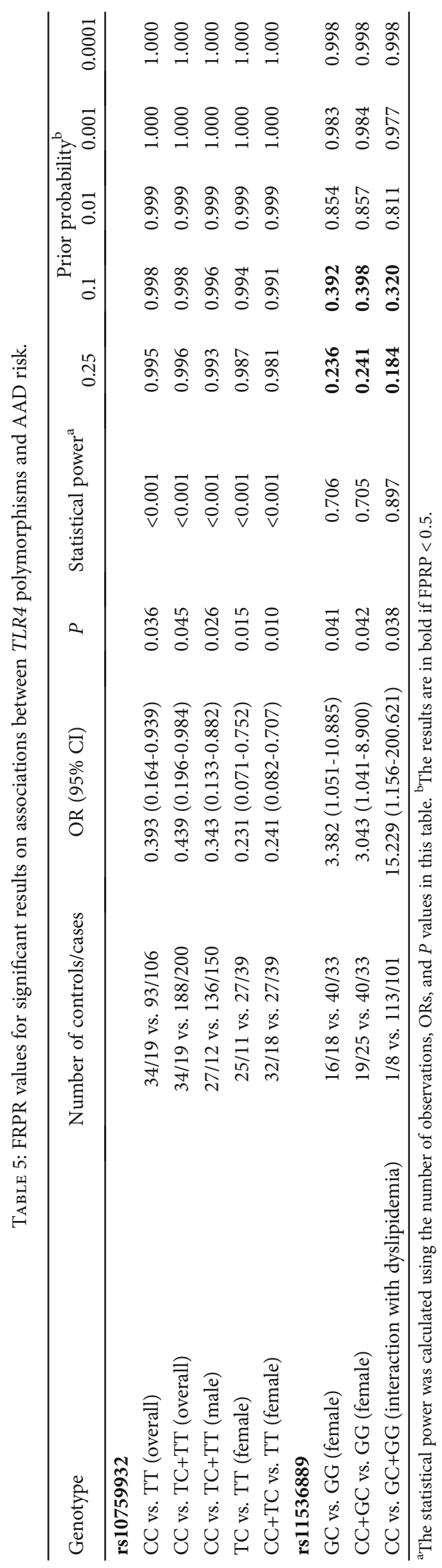


TABLE 6: Association of TLR4 polymorphisms with clinical parameters and serum TLR4 levels in AAD patients.

\begin{tabular}{|c|c|c|c|c|c|c|}
\hline Variables & $\mathrm{TT}(n=106)$ & $\begin{array}{l}\text { rs10759932 } \\
\text { TC }(n=94)\end{array}$ & $\mathrm{CC}(n=19)$ & $\mathrm{GG}(n=148)$ & $\begin{array}{l}\text { rs11536889 } \\
\text { GC }(n=58)\end{array}$ & $\mathrm{CC}(n=16)$ \\
\hline $\mathrm{WBC}\left(\times 10^{9} / \mathrm{L}\right)$ & $10.84 \pm 4.35$ & $11.63 \pm 4.39$ & $11.48 \pm 5.43$ & $11.06 \pm 4.47$ & $11.80 \pm 3.94$ & $11.07 \pm 6.12$ \\
\hline CRP (mg/L) & $77.03 \pm 65.71$ & $76.57 \pm 54.78$ & $71.58 \pm 63.35$ & $79.51 \pm 63.79$ & $72.95 \pm 55.34$ & $69.51 \pm 55.72$ \\
\hline $\mathrm{D}$-dimer $(\mu \mathrm{g} / \mathrm{mL})$ & $4.39 \pm 4.24$ & $4.68 \pm 4.49$ & $6.34 \pm 6.17$ & $4.76 \pm 4.57$ & $4.81 \pm 4.66$ & $2.95 \pm 2.40$ \\
\hline Max. aortic diameter $(\mathrm{cm})$ & $4.40 \pm 0.86$ & $4.55 \pm 1.06$ & $4.69 \pm 0.76$ & $4.40 \pm 0.82$ & $4.55 \pm 1.12$ & $4.96 \pm 1.12^{*}$ \\
\hline Serum TLR4 levels (ng/mL) & $14.83 \pm 7.25$ & $12.35 \pm 5.56$ & $9.78 \pm 6.13^{*}$ & $13.97 \pm 7.69$ & $13.07 \pm 5.69$ & $10.83 \pm 3.56$ \\
\hline
\end{tabular}

${ }^{*} P<0.05$ vs. wild-type.

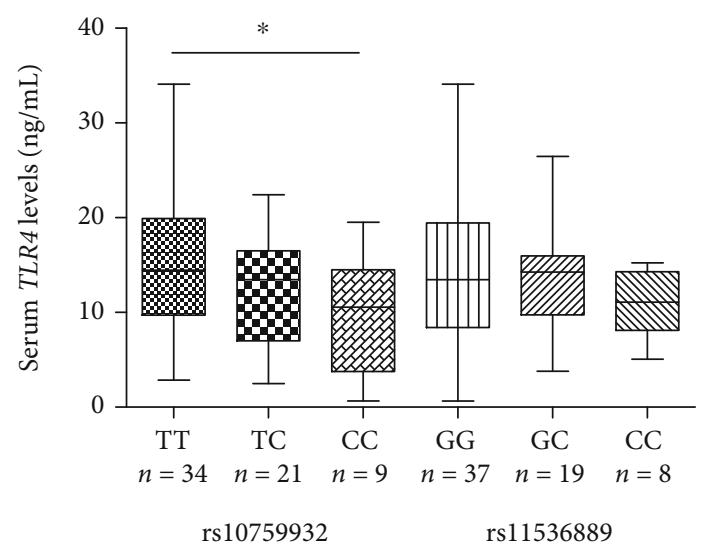

Figure 1: The effect of TLR4 rs10759932 and rs11536889 polymorphisms on serum TLR4 levels. ${ }^{*} P<0.05$.

environmental exposure [5]. We further analyzed the interaction of TLR4 rs10759932 and rs11536889 polymorphism with obesity, smoking, drinking, hypertension, and dyslipidemia in the risk of AAD. Only the effect between the rs11536889 recessive model and dyslipidemia was observed to relate to an increased risk of AAD with an OR value of 15.229 after adjusting the potential confounders. Abnormal blood lipid composition has been demonstrated to play a key role in the occurrence and development of aortic dissection [35]. Moreover, as endogenous ligands, modified lipoproteins may activate the TLR4 signaling pathway and influence the function of TLR4 $[36,37]$. Recent evidence suggested that hyperlipidemia was able to modulate TLR4 activation and contribute to the increased TLR4 expression $[38,39]$. Our findings indicated that the genetic variation along with dyslipidemia could lead to a synergistic reaction on AAD susceptibility.

Activation of TLR 4 can induce the production and secretion of proinflammatory cytokine, while AAD is frequently accompanied by a systemic inflammatory response and severe coagulopathies, which may be provoked by acute aortic injury and reflected in an increment in serum levels of WBC count, CRP, and D-dimer [8, 40,41]. The maximal aortic diameter is a well-established determinant of adverse clinical events in patients with aortic disorder. The combined application of genetic analysis and noninvasive vascular imaging holds promise for the prediction and risk stratification of $\mathrm{AAD}$ patients [42]. Interestingly, although our results showed no significant association of TLR4 rs10759932 or rs11536889 polymorphism with serum WBC, CRP, and Ddimer levels, AAD patients with rs11536889 CC genotype displayed larger aortic diameters compared to those with GG genotype. Furtherly, our genotype-phenotype analysis suggested that the CC genotype of rs10759932 linked with AAD susceptibility contributed to lower serum TLR4 levels than the TT genotype. Thus, we speculate that rs10759932 polymorphism could have a protective effect on AAD risk by downregulating the expression of TLR4. However, further molecular experiments should be carried out to verify our results.

Several limitations existed in the current study. First, because of the fact that $\mathrm{AAD}$ was a rare vascular disease, our sample size was relatively small, especially for stratification and interaction analyses, so further replication and validation in larger and different populations were required. Second, there were some missing data in demographic and clinical parameters. In addition, our investigation was a genetic association study and lacked in vitro functional confirmation tests. Therefore, a series of experiments were needed to identify the potential molecular mechanisms of the significant SNPs in the future.

\section{Conclusion}

In summary, our data demonstrated that TLR4 rs10759932 was a protective factor whereas rs11536889 was a risk factor for AAD in a Chinese Han population, and these genetic correlations were independent of the classical cardiovascular risk factors. And the interaction between rs11536889 recessive model and dyslipidemia could enhance the susceptibility to AAD. Furthermore, rs 11536889 had a significant impact on AAD size, and rs10759932 was in an evident association with serum TLR4 expression levels. Our findings may provide context for the better understanding of genetic features of AAD and thus facilitate the improvement of diagnostic and therapeutic approaches for AAD patients.

\section{Data Availability}

The data used to support the findings of this study are available from the corresponding author upon request.

\section{Conflicts of Interest}

The authors state that they have no competing interests. 


\section{Authors' Contributions}

Tan Li performed the experiments, conducted statistical analysis, and wrote the manuscript. Xiaozheng Liu and Hongxia Ning collected the blood samples. Xintong Li contributed to the collection of clinical data. Jun Yang participated in the research design. Chunyan $\mathrm{Ma}$ designed the research and revised the manuscript. All authors read and approved the final manuscript.

\section{Acknowledgments}

This study was supported by grants from the National Natural Science Foundation of China (82001828 and 81871373), the Natural Science Foundation of Liaoning Province (2020-BS102), and the Backbone Youth Support Project (Natural Science) of China Medical University (QGZ2018007).

\section{References}

[1] E. J. Benjamin, P. Muntner, A. Alonso et al., "Heart disease and stroke statistics-2019 update: a report from the American Heart Association," Circulation, vol. 139, no. 10, pp. e56e528, 2019.

[2] F. Luo, X. L. Zhou, J. J. Li, and R. T. Hui, "Inflammatory response is associated with aortic dissection," Ageing Research Reviews, vol. 8, no. 1, pp. 31-35, 2009.

[3] J. Han, J. Liu, Q. Zhou, S. Nie, J. Liu, and S. Wen, "Single nucleotide polymorphisms (SNPs) genotyping reveals that Mfn2 polymorphisms are associated with thoracic aortic dissection in Han Chinese population," Medical Science Monitor, vol. 25, pp. 2419-2428, 2019.

[4] S. A. LeMaire and L. Russell, "Epidemiology of thoracic aortic dissection," Nature Reviews. Cardiology, vol. 8, no. 2, pp. 103$113,2011$.

[5] X. L. Wang, O. Liu, Y. W. Qin, H. J. Zhang, and Y. Lv, “Association of the polymorphisms of MMP-9 and TIMP-3 genes with thoracic aortic dissection in Chinese Han population," Acta Pharmacologica Sinica, vol. 35, no. 3, pp. 351-355, 2014.

[6] F. del Porto, M. Proietta, L. Tritapepe et al., "Inflammation and immune response in acute aortic dissection," Annals of Medicine, vol. 42, no. 8, pp. 622-629, 2010.

[7] H. Kuehl, H. Eggebrecht, T. Boes et al., "Detection of inflammation in patients with acute aortic syndrome: comparison of FDG-PET/CT imaging and serological markers of inflammation," Heart, vol. 94, no. 11, pp. 1472-1477, 2008.

[8] X. Liu, G. Wang, and T. Zhang, "The analysis of the levels of plasma inflammation-related cytokines and endotoxins in patients with acute aortic dissection," Clinical Hemorheology and Microcirculation, vol. 76, no. 1, pp. 1-7, 2020.

[9] T. Suzuki, E. Bossone, D. Sawaki et al., "Biomarkers of aortic diseases," American Heart Journal, vol. 165, no. 1, pp. 15-25, 2013.

[10] C. Qin, H. Zhang, J. Gu, Z. Xiao, Q. Yang, and W. Meng, "Dynamic monitoring of platelet activation and its role in post-dissection inflammation in a canine model of acute type A aortic dissection," Journal of Cardiothoracic Surgery, vol. 11, no. 1, p. 86, 2016.

[11] N. Cifani, M. Proietta, L. Tritapepe et al., "Stanford-A acute aortic dissection, inflammation, and metalloproteinases: a review," Annals of Medicine, vol. 47, no. 6, pp. 441-446, 2015.

[12] Z. Huang, Y. Liu, L. Liang et al., “Association of toll-like receptor 4 polymorphisms with the risk of coronary artery disease in the ethnic Zhuang population of the Guangxi Province of China," Gene, vol. 708, pp. 1-9, 2019.

[13] C. R. Balistreri, "Genetic contribution in sporadic thoracic aortic aneurysm? Emerging evidence of genetic variants related to TLR-4-mediated signaling pathway as risk determinants," Vascular Pharmacology, vol. 74, pp. 1-10, 2015.

[14] C. R. Balistreri, G. Ruvolo, D. Lio, and R. Madonna, “Toll-like receptor-4 signaling pathway in aorta aging and diseases: "its double nature"," Journal of Molecular and Cellular Cardiology, vol. 110, pp. 38-53, 2017.

[15] Y. Wang, M. X. Zhang, X. Meng et al., "Atorvastatin suppresses LPS-induced rapid upregulation of toll-like receptor 4 and its signaling pathway in endothelial cells," American Journal of Physiology. Heart and Circulatory Physiology, vol. 300, no. 5, pp. H1743-H1752, 2011.

[16] C. Pisano, C. R. Balistreri, A. Ricasoli, and G. Ruvolo, "Cardiovascular disease in ageing: an overview on thoracic aortic aneurysm as an emerging inflammatory disease," Mediators of Inflammation, vol. 2017, 8 pages, 2017.

[17] Z. Wang, Z. Wang, J. Zhu, X. Long, and J. Yan, "Vitamin K2 can suppress the expression of toll-like receptor 2 (TLR2) and TLR4, and inhibit calcification of aortic intima in ApoE(-/-) mice as well as smooth muscle cells," Vascular, vol. 26, no. 1, pp. 18-26, 2018.

[18] T. Li, J. J. Jing, J. Yang et al., "Serum levels of matrix metalloproteinase 9 and toll-like receptor 4 in acute aortic dissection: a case-control study," BMC Cardiovascular Disorders, vol. 18, no. 1, p. 219, 2018.

[19] Y. Jiao, J. Zhao, B. Hu et al., "Toll-like receptor 4 gene is associated with recurrent spontaneous miscarriage in Uygur and Han women in Xinjiang," Experimental and Therapeutic Medicine, vol. 12, no. 5, pp. 3268-3274, 2016.

[20] T. Li, X. Zhang, L. Sang et al., "The interaction effects between TLR4 and MMP9 gene polymorphisms contribute to aortic aneurysm risk in a Chinese Han population," BMC Cardiovascular Disorders, vol. 19, no. 1, p. 72, 2019.

[21] T. Tongtawee, T. Simawaranon, W. Wattanawongdon, C. Dechsukhum, and W. Leeanansaksiri, "Toll-like receptor 2 and 4 polymorphisms associated with Helicobacter pylori susceptibility and gastric cancer," The Turkish Journal of Gastroenterology, vol. 30, no. 1, pp. 15-20, 2019.

[22] X. Xie, X. Shi, and M. Liu, "The roles of TLR gene polymorphisms in atherosclerosis: a systematic review and metaanalysis of 35,317 subjects," Scandinavian Journal of Immunology, vol. 86, no. 1, pp. 50-58, 2017.

[23] Y. Rupasree, S. M. Naushad, L. Rajasekhar, A. Uma, and V. K. Kutala, "Association of TLR4 (D299G, T399I), TLR9 -1486 T $>$ C, TIRAP S180L and TNF- $\alpha$ promoter $(-1031,-863$, -857) polymorphisms with risk for systemic lupus erythematosus among South Indians," Lupus, vol. 24, no. 1, pp. 50-57, 2014.

[24] K. Zhang, B. Zhou, Y. Wang, L. Rao, and L. Zhang, “The TLR4 gene polymorphisms and susceptibility to cancer: a systematic review and meta-analysis," European Journal of Cancer, vol. 49, no. 4, pp. 946-954, 2013.

[25] G. Ruvolo, C. Pisano, G. Candore et al., "Can the TLR-4mediated signaling pathway be "a key inflammatory promoter 
for sporadic TAA"?," Mediators of Inflammation, vol. 2014, 14 pages, 2014.

[26] C. He, H. Tu, L. Sun et al., "Helicobacter pylori-related host gene polymorphisms associated with susceptibility of gastric carcinogenesis: a two-stage case-control study in Chinese," Carcinogenesis, vol. 34, no. 7, pp. 1450-1457, 2013.

[27] S. Wacholder, S. Chanock, M. Garcia-Closas, L. El Ghormli, and N. Rothman, "Assessing the probability that a positive report is false: an approach for molecular epidemiology studies," Journal of the National Cancer Institute, vol. 96, no. 6, pp. 434-442, 2004.

[28] P. V. Chumachenko, A. Y. Postnov, A. G. Ivanova et al., "Thoracic aortic aneurysm and factors affecting aortic dissection," Journal of Personalized Medicine, vol. 10, no. 4, p. 153, 2020.

[29] H. Huang, J. Wu, G. Jin et al., "A 5 '-flanking region polymorphism in toll-like receptor 4 is associated with gastric cancer in a Chinese population," Journal of Biomedical Research, vol. 24, no. 2, pp. 100-106, 2010.

[30] Y. C. Chen, E. Giovannucci, R. Lazarus, P. Kraft, S. Ketkar, and D. J. Hunter, "Sequence variants of toll-like receptor 4 and susceptibility to prostate cancer," Cancer Research, vol. 65, no. 24, pp. 11771-11778, 2005.

[31] X. Zhao, J. Feng, L. Zhang et al., "One functional variant in the 3 '-untranslated region of TLR4 is associated with the elevated risk of ventilator-associated pneumonia in the patients with chronic obstructive pulmonary disease," Journal of Cellular Physiology, vol. 234, no. 10, pp. 18879-18886, 2019.

[32] K. Sato, A. Yoshimura, T. Kaneko et al., "A single nucleotide polymorphism in 3'-untranslated region contributes to the regulation of toll-like receptor 4 translation," The Journal of Biological Chemistry, vol. 287, no. 30, pp. 25163-25172, 2012.

[33] N. Castano-Rodriguez, N. O. Kaakoush, A. L. Pardo, K. L. Goh, K. M. Fock, and H. M. Mitchell, "Genetic polymorphisms in the toll-like receptor signalling pathway in Helicobacter pylori infection and related gastric cancer," Human Immunology, vol. 75, no. 8, pp. 808-815, 2014.

[34] P. Kashyap, M. Deka, S. Medhi, S. Dutta, K. Kashyap, and N. Kumari, "Association of toll-like receptor 4 with hepatitis A virus infection in Assam," Acta Virologica, vol. 62, no. 1, pp. 58-62, 2018.

[35] H. Tanaka, Y. Iida, T. Iwaki et al., "Elevated plasma levels of LDL cholesterol promote dissecting thoracic aortic aneurysms in angiotensin II-induced mice," Annals of Vascular Surgery, vol. 48, pp. 204-213, 2018.

[36] L. Chavez-Sanchez, A. Madrid-Miller, K. Chavez-Rueda, M. V. Legorreta-Haquet, E. Tesoro-Cruz, and F. Blanco-Favela, "Activation of TLR2 and TLR4 by minimally modified lowdensity lipoprotein in human macrophages and monocytes triggers the inflammatory response," Human Immunology, vol. 71, no. 8, pp. 737-744, 2010.

[37] Y. I. Miller, S. H. Choi, P. Wiesner, and Y. S. Bae, "The SYK side of TLR4: signalling mechanisms in response to LPS and minimally oxidized LDL," British Journal of Pharmacology, vol. 167, no. 5, pp. 990-999, 2012.

[38] A. Kapelouzou, S. Giaglis, M. Peroulis et al., "Overexpression of toll-like receptors $2,3,4$, and 8 is correlated to the vascular atherosclerotic process in the hyperlipidemic rabbit model: the effect of statin treatment," Journal of Vascular Research, vol. 54, no. 3, pp. 156-169, 2017.

[39] Y. J. Zhu, C. Wang, G. Song, S. S. Zang, Y. X. Liu, and L. Li, "Toll-like receptor- 2 and -4 are associated with hyperlipid- emia," Molecular Medicine Reports, vol. 12, no. 6, pp. 82418246, 2015.

[40] D. Wen, H. Y. Wu, X. J. Jiang et al., "Role of plasma C-reactive protein and white blood cell count in predicting in-hospital clinical events of acute type A aortic dissection," Chinese Medical Journal, vol. 124, no. 17, pp. 2678-2682, 2011.

[41] S. M. Yuan, Y. H. Shi, J. J. Wang, F. Q. Lu, and S. Gao, "Elevated plasma $\mathrm{D}$-dimer and hypersensitive $\mathrm{C}$-reactive protein levels may indicate aortic disorders," Revista Brasileira de Cirurgia Cardiovascular, vol. 26, no. 4, pp. 573-581, 2011.

[42] K. Kato, Y. Tokuda, N. Inagaki et al., "Association of a matrix metallopeptidase 1 gene polymorphism with long-term outcome of thoracic aortic aneurysm," International Journal of Molecular Medicine, vol. 29, no. 1, pp. 125-132, 2012. 